\title{
Processing facial expressions of emotion: upright vs. inverted images
}

\author{
David L. Bimler ${ }^{1}$, Slawomir J. Skwarek ${ }^{2}$ and Galina V. Paramei ${ }^{3}$ \\ ${ }^{1}$ School of Arts, Development and Health Education, Massey University, Palmerston North, New Zealand \\ 2 Institute for Leadership and Personal Management, University of St. Gallen, St. Gallen, Switzerland \\ ${ }^{3}$ Department of Psychology, Liverpool Hope University, Liverpool, UK
}

\section{Edited by:}

Linda Isaac, Palo Alto VA and Stanford University, USA

\section{Reviewed by:}

Daphne Maurer, McMaster

University, Canada

Glenda C. Prkachin, University of

Northern British Columbia, Canada

*Correspondence:

David L. Bimler, School of Arts, Development and Health Education, Massey University College of Education, Centennial Drive,

Palmerston North 4442, New Zealand. e-mail:d.bimler@massey.ac.nz
We studied discrimination of briefly presented upright vs. inverted emotional facial expressions (FEs), hypothesizing that inversion would impair emotion decoding by disrupting holistic FE processing. Stimuli were photographs of seven emotion prototypes, of a male and female poser (Ekman and Friesen, 1976), and eight intermediate morphs in each set. Subjects made speeded Same/Different judgments of emotional content for all upright (U) or inverted (I) pairs of FEs, presented for $500 \mathrm{ms,} 100$ times each pair. Signal Detection Theory revealed the sensitivity measure $d^{\prime}$ to be slightly but significantly higher for the upright FEs. In further analysis using multidimensional scaling (MDS), percentages of Same judgments were taken as an index of pairwise perceptual similarity, separately for $U$ and I presentation mode. The outcome was a 4D "emotion expression space," with FEs represented as points and the dimensions identified as Happy-Sad, Surprise/Fear, Disgust, and Anger. The solutions for $U$ and I FEs were compared by means of cophenetic and canonical correlation, Procrustes analysis, and weighted-Euclidean analysis of individual differences. Differences in discrimination produced by inverting FE stimuli were found to be small and manifested as minor changes in the MDS structure or weights of the dimensions. Solutions differed substantially more between the two posers, however. Notably, for stimuli containing elements of Happiness (whether $U$ or I), the MDS structure showed signs of implicit categorization, indicating that mouth curvature - the dominant feature conveying Happiness - is visually salient and receives early processing. The findings suggest that for briefly presented FEs, Same/Different decisions are dominated by low-level visual analysis of abstract patterns of lightness and edge filters, but also reflect emerging featural analysis. These analyses, insensitive to face orientation, enable initial positive/negative Valence categorization of FEs.

Keywords: facial expressions, emotion, inversion, Same/Different, signal detection theory, multidimensional scaling, categorization, featural analysis

\section{INTRODUCTION}

Facial expressions (FEs) contain information about emotional state, but despite decades of research, the nature of this information is still far from definite. Nor is it clear what stages of visual processing are involved in perception of a facial expression (FE), i.e., how face pictorial cues conveying this information are translated into a mental/affective representation. If some kind of perturbation or degradation of the stimuli selectively disrupted some aspects of facial information more than others, this would provide clues to the underlying mechanics of FE perception.

A research tradition has examined the effects of inverting FE stimuli as a simple way of disrupting their perception. An extreme possibility is that inverting FEs takes away their emotional content (e.g., Parks et al., 1985). However, examining misidentifications among inverted FEs displayed for $15 \mathrm{~s}$, McKelvie (1995) found that although these were mislabeled more often than upright FEs, they still conveyed emotions more accurately than chance would predict. The overall pattern of confusions was similar in both presentation modes, with relatively high confusion rates between particular pairs of emotions (e.g., Fear misread as Surprise and vice versa). This finding has since been replicated with briefer and with unlimited exposures (Prkachin, 2003; Calvo and Nummenmaa, 2008; Derntl et al., 2009; Narme et al., 2011). It indicates that the disruptive impact of inversion upon FE processing is not complete, and is general rather than being confined to specific expressions.

The approach taken here is to collect and analyze perceptual similarities, a well-established methodology in the FE domain. In the studies cited above, misidentifications of displayed emotions can be regarded as a behavioral index of similarity between presented FE stimuli and implicit, latent prototypes of emotional expression. Elsewhere the data consist of explicitly judged interstimulus similarities (e.g., Gladstones, 1962; Stringer, 1967; Bimler and Kirkland, 2001). Here the similarities take the form of another behavioral measure: the probability of wrongly identifying two (similar) FE stimuli as duplicates in a speeded Same/Different $(S / D)$ task. $S / D$ errors have been used as a surrogate for similarity in several studies of FE categorical perception, in order to locate the 
boundary between two emotion categories (e.g., Calder et al., 1996, Experiment 4; Roberson et al., 1999) ${ }^{1}$. Our analysis focuses on a comparison between the overall structure of similarities among inverted FEs and the similarities among the same stimuli when they are upright. The comparison uses several complementary metrics to quantify any upright/inverted difference.

The challenge in the analysis of similarities is to extract any clues the raw data might contain about perceptual processing, and in particular, about the level of processing accessed when determining similarity. In themselves, similarity data do not speak directly to the presence of emotional content after inversion, but any evidence that the stimuli are perceived categorically could be taken as a sign of the presence of emotional categories.

At one extreme, subjects might base their FE comparisons upon the visual images in a relatively raw form, i.e., derivativeof-Gaussian edge filters (Marr, 1982; Dailey et al., 2002), where inversion would have little impact. Conversely, later stages of visual processing would be involved if the comparisons draw upon cues or information extracted from FE stimuli: in particular, featural and/or configural information ${ }^{2}$. If both forms of information are extracted, and one is more accessible when faces are upright, then inverting the stimuli will reduce the contribution of these cues to perceived inter-stimulus dissimilarity.

There is evidence from a closely related perceptual domain that of facial identity and recognition - that inversion selectively disrupts configural (second-order) cues. Inversion impairs face recognition in a qualitative rather than quantitative way (Yin, 1969; Leder and Bruce, 2000). In comparison, analysis of featural information is disrupted to a lesser degree (Farah et al., 1995; Maurer et al., 2002). The question, then, is whether configural cues are equally important in processing FEs.

The configural aspect of a face consists of how its features are arranged; it is synonymous with "relational" or "spatial-relational" (Diamond and Carey, 1986; Bartlett and Searcy, 1993). Conversely, the featural aspect consists of local descriptions, or feature-specific cues to emotional state, e.g., mouth curvature, eye openness, or eyebrow lowering.

It has been argued that "the various expressions reflect spatial relationships and distinctive features to different degrees" (McKelvie, 1995, p. 332). If so, an empirical answer is possible because disruption of configural cues will selectively impact on the transmission of some emotions more than others (Smith and Schyns, 2009). Disgust is arguably a configural, "holistic" expression, since local featural correlates for it are elusive (Paramei and Benson, 1998; Bimler and Paramei, 2006). In comparison, Happiness and Anger are "featural," relatively "localized" emotions, expressed by the lower and upper half of a FE respectively, whereas Surprise is expressed with equal force by both halves (Bassili, 1979; Calder

\footnotetext{
${ }^{1}$ In addition to accuracy, we also measured response times, RTs, taken to correctly recognize two stimuli as non-identical and used the median RTs for each stimulus pair as a second index of similarity. Our RT analysis reinforces the current conclusions (Paramei et al., 2009), but it exhibits additional features that lie outside the scope of this report.

${ }^{2}$ These two alternatives "involve visual processing of facial stimuli at different levels of increasing perceptual complexity" (Calvo and Nummenmaa, 2008, p. 472). In a more recent account these authors differentiate perceptual, categorical and affective processing stages of FEs (Calvo et al., 2012).
}

et al., 2000; Bimler and Paramei, 2006; Fiorentini and Viviani, 2009) or the lower face (Smith and Schyns, 2009).

In exploring similarities among upright and inverted FEs, the present study follows a number of precedents by representing FEs as points in a spatial model - a multidimensional "map" - so that geometrical distances between points summarize and approximate the corresponding (dis-)similarities. The map's dimensions are identified as the affective continuous qualities on which an expression can vary. The algorithms for this form of analysis come under the rubric of multidimensional scaling (MDS). To address the putative effect of image inversion on the extraction of configural cues, we use the particular form of MDS, weighted-Euclidean individual differences MDS (Kruskal and Wish, 1978).

This approach attempts to account for multiple sets of similarities by assuming that they all arise from a single "map," while allowing the salience (weight) of some dimensions to vary according to conditions (here: upright vs. inverted), so as to optimize the match between reconstructed inter-stimulus distances and the corresponding dissimilarities in each condition. If inversion were to selectively reduce the salience of one specific dimension, this could be accommodated by compressing the map, reducing the distance between pairs of stimuli that are separated along that direction. A negative finding, i.e., no difference in FE space from inverting the stimuli, would not prove that similarity judgments are determined by featural cues in isolation. As already noted, the judgments may use information from an earlier stage of visual processing.

The spatial metaphor for FEs is only an approximation; an alternative account uses a metaphor of discrete clusters instead (e.g., Calder et al., 1996). It has been argued, though, that both forms of structure may co-exist in FE-similarity data (Dailey et al., 2002; Bimler and Paramei, 2006), much as color perception displays categorical structure as well as a dimensional "color space." Categorical perception (CP) for FEs is well-attested (e.g., Ekman and Friesen, 1976; Etcoff and Magee, 1992; Calder et al., 1996; Young et al., 1997; Bimler and Kirkland, 2001; Bimler and Paramei, 2006), with the effect of partitioning FE space into categories of prototypical FE of emotion such as of Surprise, Happiness, etcetera.

The possibility of $\mathrm{CP}$ can be explored here because the stimuli include, along with prototypical FEs, ambiguous, intermediate expressions produced by image-interpolation. Thus it is of interest whether the present data exhibit any signs of categorical processing for upright and inverted FEs. To the extent that the data show $\mathrm{CP}$ for upright or inverted stimuli, one could argue that perceptual processes have implicitly reflected the emotional content of the stimuli, enough to classify FEs by category. We should be prepared, however, for the possibility that the present conditions of brief stimulus exposure and speeded decisions will negate $\mathrm{CP}$ for both orientations.

In general, $\mathrm{CP}$ can be considered as a non-constant (sigmoidal) relationship between the objective physical dissimilarity between stimulus pairs and the corresponding perceptual dissimilarity. The physical interval between two stimuli can be small yet produce a disproportionately large dissimilarity if they straddle a category boundary. The latter is estimated using morphed stimuli interpolated at regular intervals along a perceptual continuum, between emotion prototypes of (for instance) Happy and Angry. 
Generally a category boundary emerges somewhere along the gradient. Discrimination tasks, ABX (e.g., Calder et al., 1996; de Gelder et al., 1997; Roberson and Davidoff, 2000) or XAB (e.g., Roberson and Davidoff, 2000), using morphed FEs showed that the error rate and the RTs - two measures of similarity between adjacent stimuli - both dip sharply for some interval along the gradient, coinciding with a transition in the verbal labels assigned to the morphs, which might be consistently identified as Happy on one side of the transition and as Angry on the other. Other studies used the $S / D$ task with adults (e.g., Calder et al., 1996, Experiment 4; Roberson et al., 1999, Section 5.2; Shibui et al., 2001) or, with 7-month-old infants, a novelty-preference procedure (Kotsoni et al., 2001), and obtained similar results. It appears that all these tasks are tapping into the high-level processes required to extract affective categories from the stimuli.

Retention of CP in inverted FEs is currently controversial. Inversion appears to weaken or remove category boundaries along continua of morphed stimuli [de Gelder et al., 1997, Experiment 2); Roberson et al., 1999, Section 5.2)]. However, it has no effect when an emotion category is identified and prototypical FEs are employed [ $S / D$ in a visual search task (Lipp et al., 2009); identification of a FE as "happy" or "not happy" (Calvo et al., 2012)].

Categorization, or high-level processing extracting semantic aspects of face perception, is argued to draw upon configural information (de Gelder et al., 1997). More recently, however, it was proposed that categorical processing can be feature-based - for Happy expressions with the salient mouth curvature feature and, thus, precede affective attribution at the stage of configural processing (Calvo et al., 2012).

One can hence expect FE inversion to obviate any effect of $\mathrm{CP}$, in contrast to featural cues with their relative insensitivity to orientation (reviewed by Maurer et al., 2002). The presence or absence of an effect from inversion, therefore, is a test of the proposition that configural content plays a dominant role in decoding emotional content of FEs.

By comparing performance for upright and inverted FEs in the $S / D$ task, we hope to gain insight into the cognitive mechanics of "low-level"/"high-level" processing, i.e., into "[the] cognitive or functional primitives underlying the perception of FE" that operate at "the locus of emotion perception in the cognitive architecture of the organism" (de Gelder et al., 1997, p. 20).

\section{MATERIALS AND METHODS SUBJECTS}

Two male and two female undergraduate psychology students, aged 21-25 years old, were paid to participate in 301-h-long sessions. Informed consent was obtained from all subjects. Gender of FE poser and subject gender were counterbalanced: stimuli from the "MO series" (female poser) were presented to one female subject (DK) and one male (HK); likewise, the "WF series" (male poser) was presented to one female subject (SB) and one male (BF).

\section{STIMULI}

Fourteen monochrome photographs of emotional expressions were selected from Pictures of Facial Affect (Ekman and Friesen, 1976). Ekman and Friesen deemed these 14 images to be good examples of seven universal emotion categories (Happiness, Surprise, Anger, Sadness, Fear, Disgust, Neutral), as evinced by high accuracy of labeling. Seven images featured a female poser MO while the other featured a male poser WF.

The "MO series" and "WF series" were both extended by using image-interpolation software (Design Studio) to create eight intermediate stimuli, each lying midway along the continuum defined by two emotion exemplars as end-points. Briefly, the "morphing" process involves locating "landmarks" within each prototype. Each triangle defined by three adjacent landmarks in one face can then be transformed smoothly into its counterpart into the other face, allowing stages along the transformation to be interpolated (e.g., Calder et al., 1996; Young et al., 1997). Figure 1 shows the stimuli of both series, where for clarity they have been arranged in a distorted version of the circumplex model (Russell, 1980).

These digitalized stimuli were presented on a $19^{\prime \prime}$ CRT-monitor (V7 N110s), where each image occupied $12.8 \times 8.7 \mathrm{~cm}$ (subtending $10^{\circ} \times 6.7^{\circ}$ at a viewing distance of $\left.74 \mathrm{~cm}\right)$. Measured with a LMT L1009 Luminance Meter, image luminance ranged from 0.23 to $82 \mathrm{~cd} / \mathrm{m}^{2}$. Ambient lighting in the test room was in the mesopic range (around $10 \mathrm{~cd} / \mathrm{m}^{2}$ ).

\section{PROCEDURE}

Each trial consisted of the simultaneous presentation of two FE stimuli, symmetrically side-by-side on the screen with a $3.8 \mathrm{~cm}$ gap between them (subtending $3^{\circ}$ ). After $500 \mathrm{~ms}$, the screen went blank until the subject responded via a two-button keyboard, by pressing the right button "if the two emotions are the Same" or the left button if the stimuli were Different. Subjects were instructed to respond as quickly and correctly as possible, and to press the buttons with their right and left index fingers respectively. A MS-DOS program (running on a Windows-98 PC) controlled presentations and recorded the response, Same or Different (as well as RT, from the appearance of the FE pair to the response). Each response was followed by an inter-stimulus interval of 300$400 \mathrm{~ms}$, while a small red fixation cross was displayed on the monitor.

In a single run, all possible $15 \times 15=225$ pairings of FEs were presented in randomized order. Note that 15 of these pairs were indeed identical. Within a single run, all pairs were either upright (U) or inverted (I). Runs alternated between upright and inverted stimulus pairs. For each subject the experiment began with a practice session of one run in each of the $\mathrm{U}$ and I modes. There followed ten sessions containing six runs and 20 containing seven runs, totaling to 100 runs with upright pairs and 100 in the inverted mode. These 30 sessions were spread over 4 months including a 2-month break.

Subjects were not led to expect a low proportion of samestimulus pairs or a high proportion either. Their instructions included no indication of what proportion to expect.

\section{ANALYSIS}

\section{Signal detection theory}

Signal Detection Theory (SDT) has been applied previously to analyze FE discrimination (Prkachin, 2003; Maxwell and Davidson, 2004; Milders et al., 2008; Smith and Schyns, 2009; Narme et al., 2011). Here we used SDT to analyze the correct recognition 


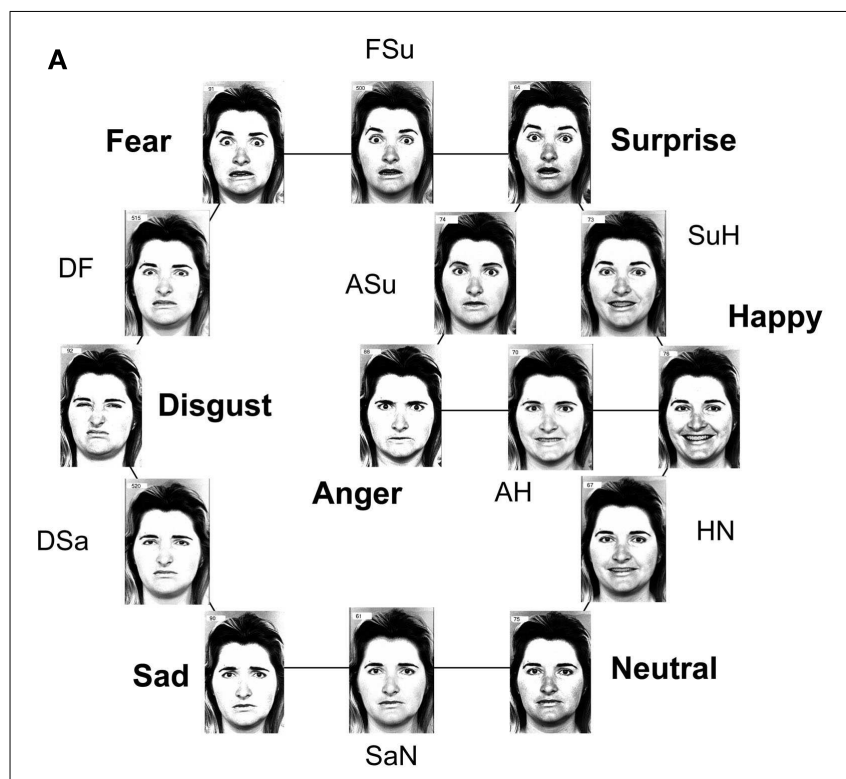

B

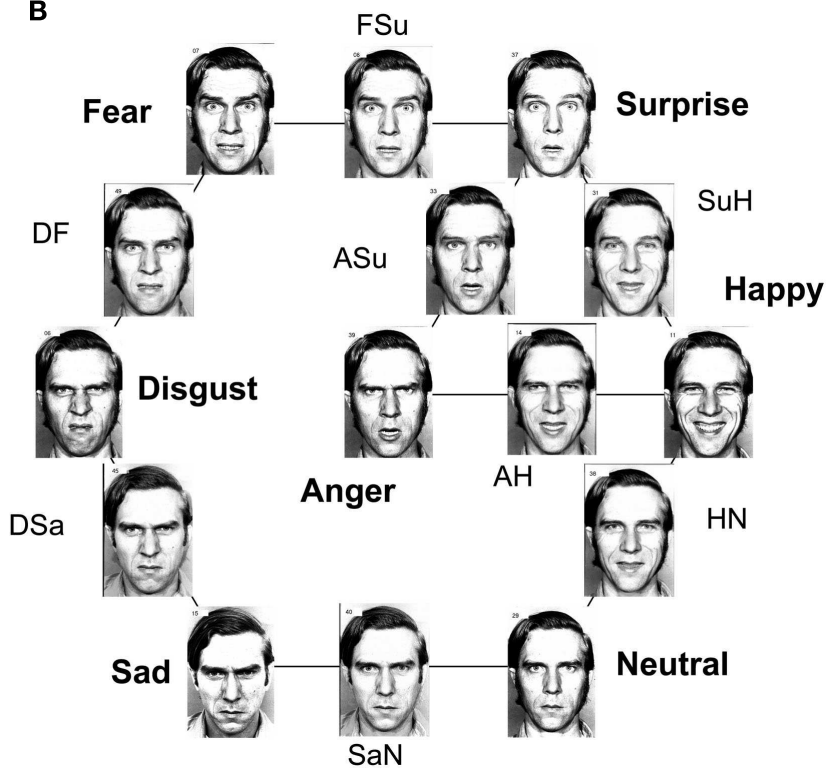

FIGURE 1 | FE stimuli, arranged in a distorted circumplex. The prototype FEs are accompanied by emotion names, while abbreviations accompany the intermediate morphs, e.g., SuH to indicate the morph between the Surprise and Happiness prototypes. (Note: circumplex arrangement is chosen for convenience, and not to indicate the similarity relationships among the seven emotional prototypes). (A) MO series; (B) WF series.

of 15 identical pairs and the erroneous Same responses to 210 different-expression pairs. For each subject, SDT yielded the sensitivity measure $d^{\prime}$ and the response criterion $C$ (Green and Swets, 1966) for each of the 100 runs, allowing these indices to be plotted against the course of data collection.

To adumbrate the Section "Results," we remark here that most different-expression pairs received a sufficiently high percentage of Same responses to rise above the statistical shot-noise. In a preliminary analysis, these "\%Same" values were examined for any obvious systematic dependence on presentation mode, i.e., whether inter-stimulus differences were more or less evident in $\mathrm{U}$ than in I mode.

\section{MDS: comparison of solutions for upright vs. inverted facial expressions}

If $A$ and $B$ are two 15-by-15 stimulus matrices of \%Same values for a given observer and presentation mode, a simple index of similarity between them is $r_{A B}$ (the bivariate correlation between corresponding entries in $A$ and $B$ across all 225 pairs of FEs). An 8 by- 8 ( 4 subjects $\times 2$ presentation modes) table of $r_{\mathrm{AB}}$ for all pairs of $A$ and $B$ was examined, to search for obvious effects of inversion and to identify those matrices which exhibit a similar underlying structure.

We went on to treat these \%Same values as estimates of the similarity between pairs of stimuli (cf. Calder et al., 1996, Experiment 4; Roberson et al., 1999), and to analyze each similarity matrix with non-metric MDS to represent and summarize its structure. The PROXSCAL MDS algorithm (implemented within SPSS) was used for this purpose.

The data proved to be sufficiently robust that separate MDS solutions could be fitted to each \%Same matrix, i.e., to each combination of subject, poser, and presentation mode. To provide a common framework for qualifying the impact of inversion on perceptions of similarity, $U$ and I mode data were examined separately for each stimulus set, using the "repeated measures" feature of MDS to combine the \%Same matrices from two subjects into a single configuration. Pooling data also increases the accuracy of the solutions. This led to four MDS solutions, which can be labeled MO-U, MO-I, WF-U, and WF-I.

We quantified the concordance between pairs of MDS solutions, U vs. I, in three complementary ways to avoid the limitations of any single metric for comparison.

(a) Cophenetic correlation. This is the correlation (c) between each inter-point distance in the $U$ solution and its counterpart in the I solution;

(b) Procrustes analysis consists of superimposing the U and I solutions, rotating and rescaling them to minimize the total distance $\left(g_{l}\right)$ between corresponding pairs of points ( $g_{l}$ drops to 0 if the solutions are geometrically congruent and the points coincide after rotations and rescaling);

(c) Canonical correlation or CANCORR extracts a pair of linear combinations from the $\mathrm{U}$ and I coordinate sets, such that the correlation $R_{\mathrm{c}}$ between them is maximal. It can extract further pairs of linear combinations of coordinates, providing correlations $R_{2}, R_{3}, R_{4}$ (each new combination being orthogonal to those previously extracted from its respective coordinate set). The number of significant correlations indicates the number of mutually recognizable dimensions shared between the two coordinate sets.

CANCORR is blind to possible differences in the salience of shared dimensions. With this in mind, Weighted-Euclidean model of individual differences was used to quantify the effect of inversion, analyzing responses for $\mathrm{U}$ and I stimuli in conjunction. This required two "group configurations," one for each of the MO 
(female) and WF (male) series, and each based on four (2 subjects $\times 2$ presentation modes) data matrices. Locations of points in these configurations were then held constant, while optimizing the fit to the data by adjusting the dimensional-weight parameters $\left(w_{i}\right)$ for each subject and presentation mode.

\section{RESULTS}

\section{SDT}

The details of the procedure used here mean that only $15 / 225=7 \%$ of trials involved physically identical stimuli. However, the subjects frequently gave a Same judgment to different-stimulus pairs as well, resulting in Same responses for about 25\% of the trials. For both presentation modes, the percentage of "false alarms" (different-expression pairs misidentified as Same) was highest for subject SB (24.3\% of U pairs and $27.3 \%$ of I pairs; the difference being significant at $p=0.005)$. In comparison, for $\mathrm{DK}$, the corresponding values were 16.0 and $16.3 \%$; for $\mathrm{HK}, 19.4$ and $17.3 \%$ (a significant difference at $p<0.001$ ); for $\mathrm{BF}, 15.8$ and $16.0 \%$. This over-vigilance toward "sameness" and willingness to accept false positives is reflected in consistently negative values of the response criterion $C$ for all subjects across the course of the experiment (see Figure A1 in Appendix) $)^{3}$.

The sensitivity measure $d^{\prime}$ tends to be slightly higher in the U than in the I mode (Figure A1 in Appendix): that is, different pairs were more distinct from identical pairs when they were presented upright. Plotting $d^{\prime}$ against experimental run reveals fluctuations from one run to the next, but no obvious evidence of a systematic increase across the course of data collection.

The impact of inversion is also evident when analyzing individual expressions (see Figure 2). Each of the 15 FEs was presented 100 times as an identical-expression pair while 2800 presentations paired it with a different-expression. For each FE, the analysis combines the corresponding rates of Same responses. Figure 2 reveals a general trend for inversion to reduce the sensitivity measure $d^{\prime}$ (more points are below the diagonal), significantly so for three subjects (one-sided Wilcoxon test): $p=0.024$ for $\mathrm{DK}, 0.003$ for $\mathrm{HK}, 0.01$ for BF, compared to 0.36 for SB.

No consistent inversion effect can be discerned, however, affecting any specific FE prototype or its morphs more than the others. Details among the stimuli vary between the two posers, MO and WF. For the former, $d^{\prime}$ was highest for Happiness, Surprise, Fear, $F S u$, and Disgust (i.e., the difference is relatively easy to detect when these expressions are paired with others) and lowest for Neutral, Sadness, SaN, and ASu. For the WF series, $d^{\prime}$ was highest for Happiness, Surprise, Anger, Sadness, and SaN, and lowest for Neutral, Fear, and $A S u$.

Although the bias toward Same responses slightly differed between the two presentation modes, these differences were minor compared to the inter-individual variations. This can be seen in Figure 3, which for individual subjects plots \% Same responses for each FE pair when seen inverted, against \%Same for the same pair in the upright mode. A systematic inversion effect would appear as an overall departure from the main diagonal toward the upper left or lower right. Accordingly, HK's data reveal that he was slightly

${ }^{3}$ Subject SB, as well as applying the criteria of "sameness" most loosely, also responded more slowly than the other subjects by about $300 \mathrm{~ms}$.
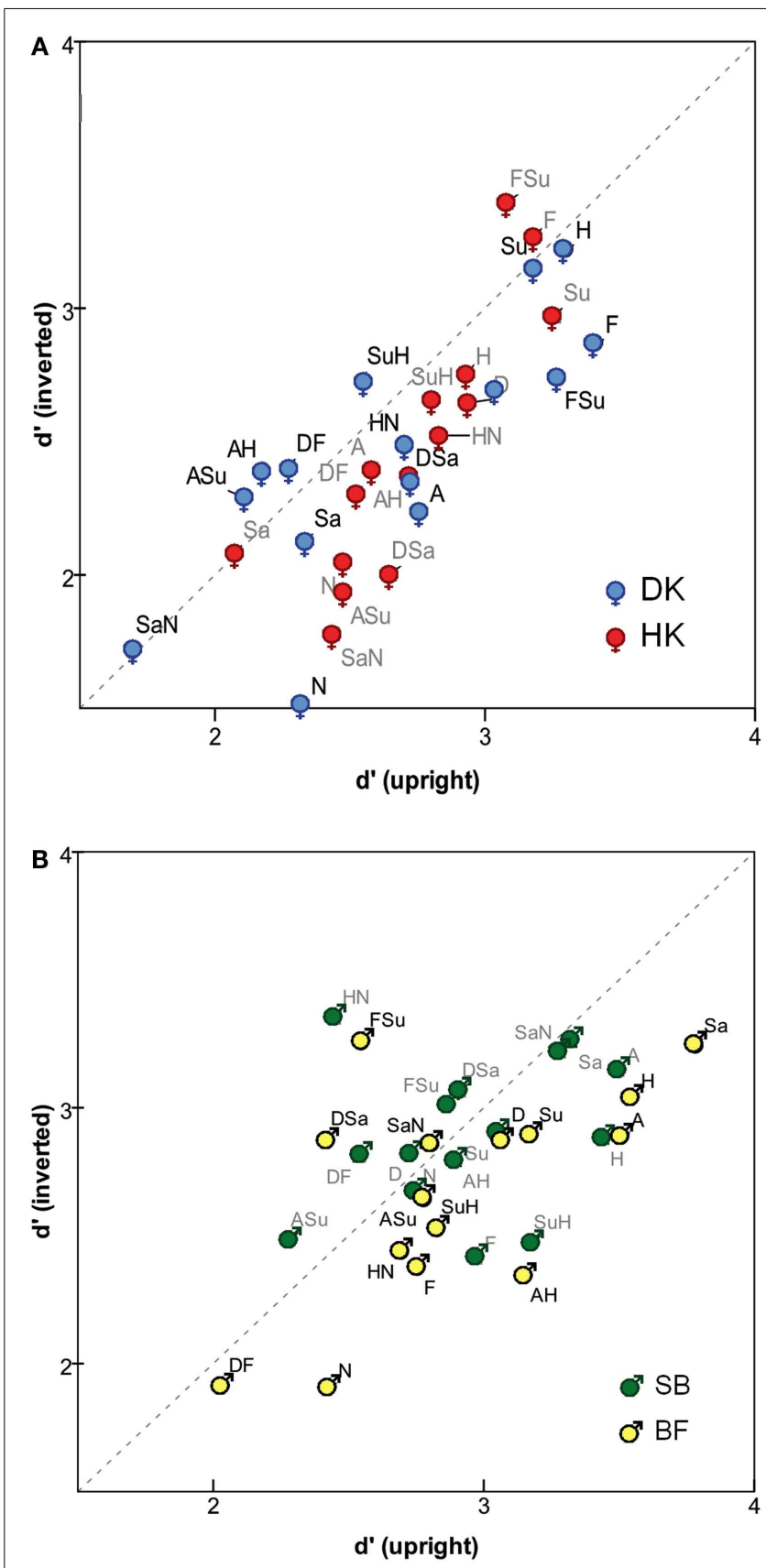

FIGURE 2 | Sensitivity measure $d^{\prime}$ for identifying identical pairs of specific expressions, in upright mode (horizontal axis) and inverted mode (vertical axis), for (A) MO series (subjects DK and HK), and (B) WF series (subjects BF and SB). Symbols $\uparrow=M O$ and $\hat{\delta}=W F$ indicate sex of poser, not of observer.

more likely to judge a given pair of FEs as Same when they were upright (i.e., inversion of the stimulus pair made any difference slightly more evident to him). Conversely, SB was more likely to judge an inverted pair as Same.

Figure 3 also shows a concentration of \% Same values less than $20 \%$ from glaringly different FE pairs, but the distribution was 


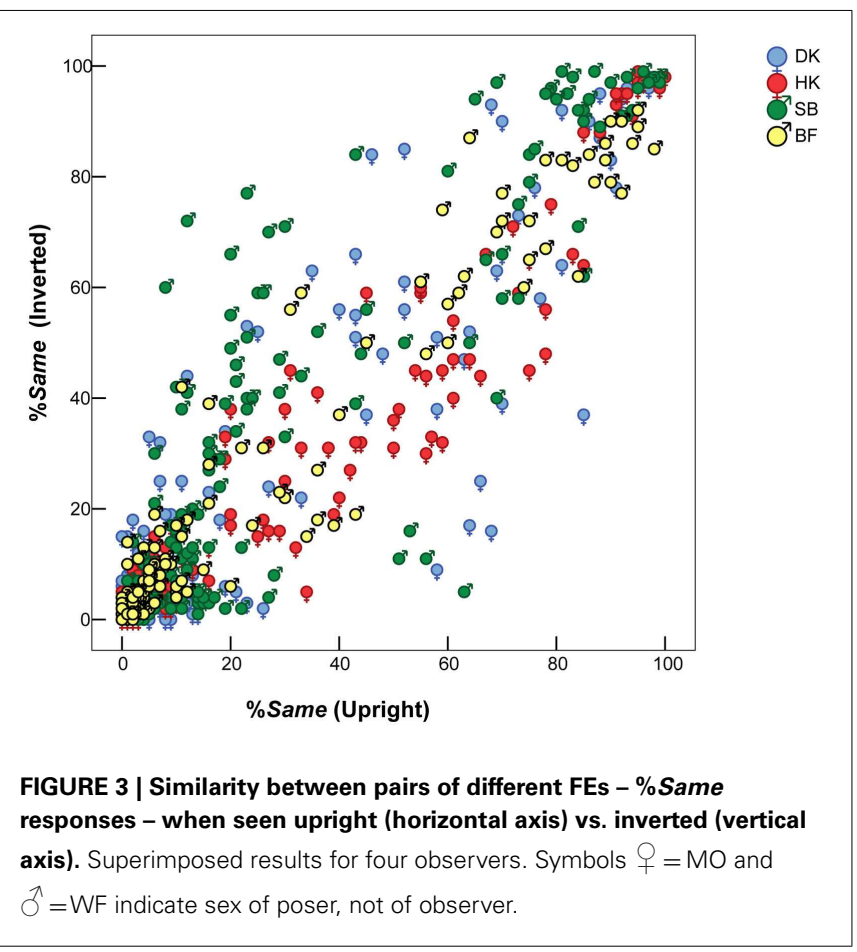

not polarized. That is, responses were probabilistic, rather than over-learned and deterministic with a consistent Same response for some pairs and a Different response for others.

\section{MDS}

\section{\%SAME MATRICES: DATA-SET CORRELATIONS}

Correlations $r$ between the eight data matrices are tabulated in Table 1. The dominant feature is a distinction between the MO and WF series of expressions, attributable to stylistic variation within an "expression prototype": posers can differ in the underlying muscle movement involved in an expression, without affecting the emotional message (Bimler and Kirkland, 2001; Alvarado and Jameson, 2002). In addition, even if two posers contract equivalent muscles to express an emotion, they may differ enough in facial structure for these to produce different results.

Table 1 also indicates that if there is any systematic difference between upright and inverted FEs, this is obscured by the larger differences among subjects. For instance, the pattern of judgments for inverted FEs from subject BF is more similar to the pattern for upright FEs from the same subject, than to the pattern for inverted FEs from subject SB.

\section{SOLUTION DIMENSIONALITY AND VARIABILITY}

We examined separate MDS solutions MO-U, MO-I for the MO (female) images and WF-U, WF-I for the WF (male) images, both when upright and inverted. A common rule-of-thumb for MDS is that the dimensionality of a solution should not exceed $N / 4$, where $N$ is the number of items (here $N=15$ ), but the limit can be relaxed in this situation where multiple data matrices are pooled. The choice of how many dimensions to retain is based on criteria such as the number of interpretable axes and the badness-of-fit values $\left(\right.$ Stress $\left._{1}\right)$. In all four cases (MO-U, MO-I, WF-U, WF-I),
Table 1 | Correlations between individual data matrices.

\begin{tabular}{llllllll}
\hline & DK-I & HK-U & HK-I & BF-U & BF-I & SB-U & SB-I \\
\hline DK-U & 0.939 & 0.935 & 0.930 & 0.357 & 0.345 & 0.313 & 0.235 \\
DK-I & & 0.947 & 0.966 & 0.382 & 0.374 & 0.338 & 0.252 \\
HK-U & & & 0.977 & 0.339 & 0.331 & 0.299 & 0.256 \\
HK-I & & & & 0.363 & 0.353 & 0.330 & 0.275 \\
BF-U & & & & & 0.981 & 0.910 & 0.803 \\
BF-I & & & & & & 0.929 & 0.829 \\
SB-U & & & & & & & 0.921 \\
\hline
\end{tabular}

$U$ - upright; I - inverted presentation mode. DK, HK, BF, and $S B$ are individual subjects.

four dimensions appeared to be optimal, yielding Stress 1 values of $0.048,0.039,0.061$, and 0.076 . These were substantial improvements on the values for three dimensions $(0.080,0.067,0.102$, and 0.103 respectively).

The robustness of these solutions was demonstrated by comparing them with our earlier four-dimensional MDS solutions for the same posers, MO and WF, derived from sorting-data for upright mode (Bimler and Paramei, 2006). Those solutions included 39 additional morphed items (i.e., 54 stimuli in total), though here we focus only on the coordinates of the 15 items included in the current set.

According to CANCORR, all four dimensions in the present FE spaces have recognizable, independent counterparts in the sorting-data solutions. In particular, in the two MO solutions all four canonical correlations were significant at $p \leq 0.002$ ( $\chi^{2}$ test on Wilks' $\Lambda$ statistic), inter-item distances are highly correlated $(r=0.84)$, and the locations of points are very similar (Procrustes distance $\left.g_{1}=0.034\right)$. In the two WF solutions, present and based on sorting-data, all four dimensions again have recognizable counterparts: the four canonical correlations were all significant at $p \leq 0.039)$; inter-item distances are very similar $(r=0.78)$, as are point locations $\left(g_{1}=0.059\right)$. This convergence is evidence that the solutions are stable, although each is based on only two data matrices.

\section{COMPARISON OF SOLUTIONS FOR UPRIGHT VS. INVERTED FACIAL EXPRESSIONS}

Table 2 shows comparisons among these separate solutions, with cophenetic correlations $c$ (above the diagonal) and Procrustes distances $g_{1}$ (below the diagonal). The high correlation and low $g_{1}$ show that very similar spaces represent MO-U and MO-I, and again for WF-U and WF-I. According to CANCORR, all four dimensions of $\mathrm{MO}-\mathrm{U}$ have recognizable, independent counterparts in MO-I: the smallest canonical correlation is $R_{4}=0.988$, and all four are significant at $(p \leq 0.001$, $\chi^{2}$ test on Wilks' $\Lambda$ statistic). All four dimensions of WF-U have counterparts in WF-I, with canonical correlations ranging down to $R_{4}=0.928(p \leq 0.001)$. It appears that inversion has had no gross effect on the subjects' ability to recognize whether two stimuli were the same or different. Given this level of convergence, we omit a detailed scrutiny of the individual solutions. 
Table 2 | Cophenetic correlations $c$ (above the diagonal) and Procrustes distances $g_{\mathrm{l}}$ (below the diagonal) between 4D MDS solutions, each based on data matrices from two subjects judging FEs of the same poser, MO or WF. U - upright, I - inverted presentation mode; MO - female poser, WF - male poser.

\begin{tabular}{lllll}
\hline & MO-U & MO-I & WF-U & WF-I \\
\hline MO-U & - & 0.93 & 0.78 & 0.74 \\
MO-I & 0.006 & - & 0.80 & 0.74 \\
WF-U & 0.047 & 0.051 & - & 0.95 \\
WF-I & 0.051 & 0.058 & 0.010 & - \\
\hline
\end{tabular}

\section{WEIGHTED-EUCLIDEAN MDS ANALYSIS}

A "group configuration" was constructed for both stimulus sets - a compromise or consensus combining four data matrices (2 subjects and 2 presentation modes) - as a pre-requisite for testing whether inversion affects the weight (salience) of the dimensions of FE space. Judging from the Stress ${ }_{1}$ values for two, three and four dimensions, a $4 \mathrm{D}$ solution was optimal for both modes of presentation and both sets.

This is a convenient point to discuss various features of these maps of FE space. After minor rotation, all four axes lend themselves to straightforward interpretations as continuous affective dimensions. D1 is a bipolar "Valence" dimension, running from Sad at its negative extreme up to Happy (and the part-Happy morphs) at the positive extreme. D2, D3, and D4 are unipolar axes of Surprise/Fear, Disgust, and Anger respectively. Two views of the $\mathrm{MO}$ solution are shown in Figure 4. In the same way, Figure 5 depicts the WF solution.

The dimensional-salience parameters $w_{i}$ from weightedEuclidean MDS are listed in Table 3. These optimize the fit between the group configurations, and subjects' responses under both modes of presentation. DK seems to be more attuned to the Disgust axis (D3) in upright stimuli; the other subjects do not exhibit this axial difference.

\section{ASSESSING CATEGORICAL PROCESSING FROM THE MDS SOLUTIONS}

Suggestions of categorical processing can be seen in Figures 4 and 5 , for stimuli containing any element of Happiness. Specifically, the Surprise/Happy (SuH), Angry/Happy (AH), and Neutral/Happy $(\mathrm{NH})$ stimuli are all close to one other and to prototypal Happiness, with a gulf between them and the other stimuli. In other words, the perceptual difference between no smile and $50 \%$ of a smile is considerably greater than the difference between $50 \%$ of a smile and a complete smile, implying that even a $50 \%$ smile is enough to reach ceiling level on our ability to detect that particular form of mouth curvature as conveying Happy emotion.

This impression can be quantified by defining a crude "Categorization Index" (CI). If $d_{1}$ is the distance in the MDS solution between a FE morph and one of its "parents," and $d_{2}$ is the distance to the second parent, then $\mathrm{CI}=d_{1} /\left(d_{1}+d_{2}\right)$. In the absence of CP, the morph would be located midway between the two prototypeexpression points (ignoring the influence of randomness in the data), and $\mathrm{CI}=0.5$. If the morph is perceptually identical to the first or the second "parent," then CI reaches its extreme values of 0 or 1 respectively.

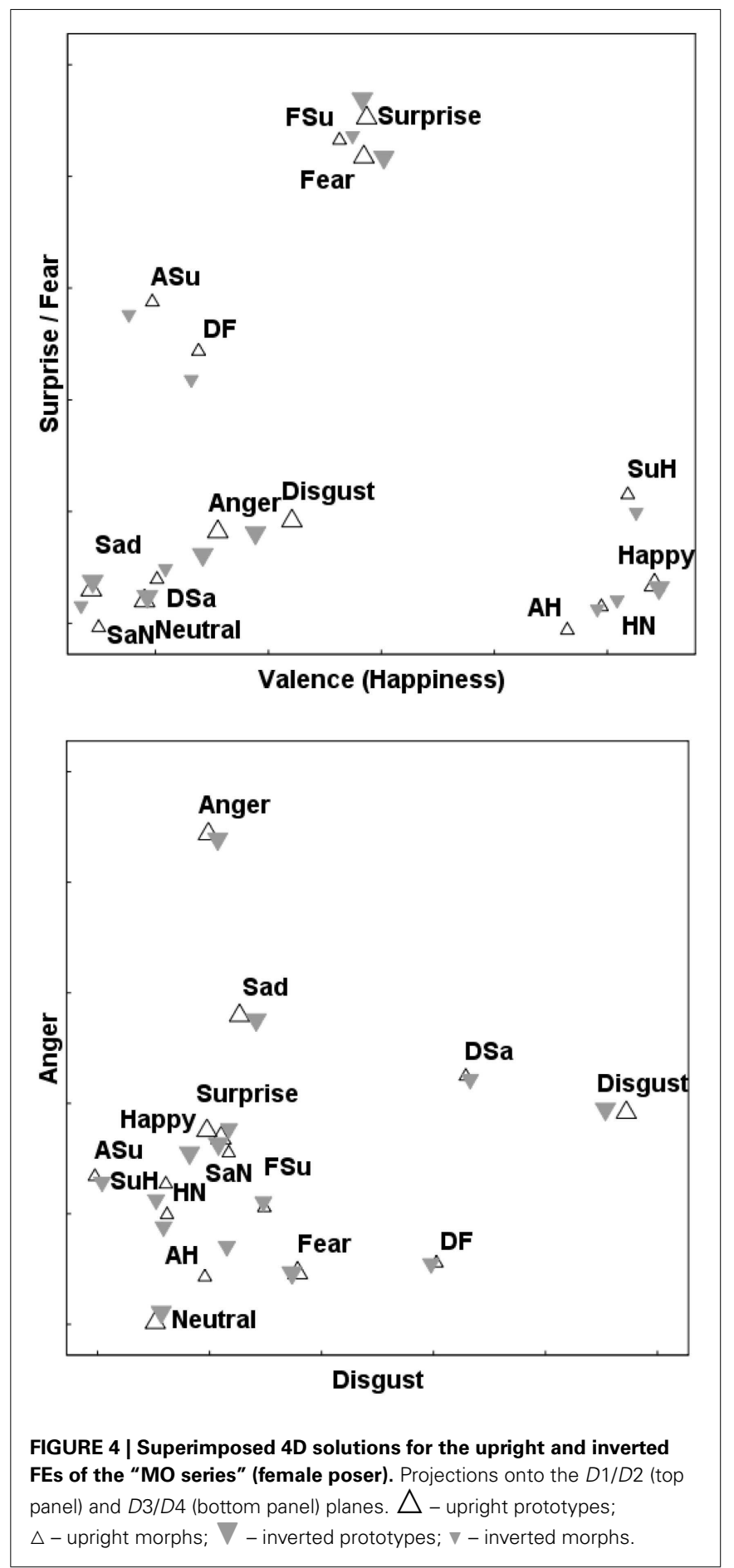

Table 4 lists CI for each of the eight morph stimuli, within the combined 4D solutions for each poser (Figures 4 and 5). In addition, CI was calculated for the locations of stimuli in the separate upright and inverted solutions. The CI information presented in Table 4 for these six solutions appears visually in Figure 6, where the six estimates of CI for each morph are shown by the location of symbols along a line between its "parent" stimuli. Figure 6 shows consistent departures from 0.5 for the three Happiness morphs. 

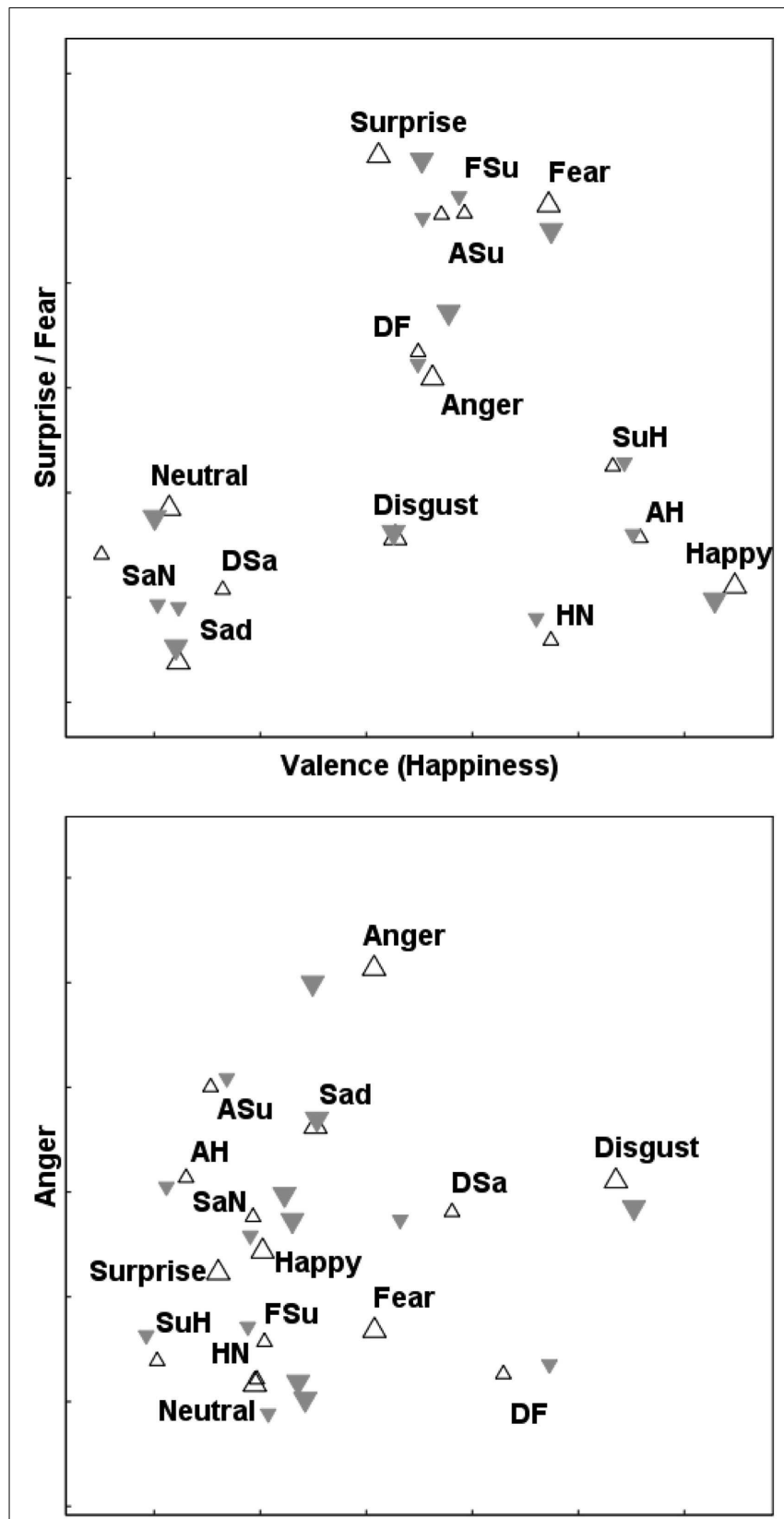

Disgust

FIGURE 5 | Superimposed 4D solutions for the upright and inverted FEs of the "WF series" (male poser). Projections onto the D1/D2 (top panel) and D3/D4 (bottom panel) planes. Symbols as in Figure 4

Note that there is no indication that departures are any weaker for the inverted mode. Signs of CP are also apparent for the Sadness/Neutral ( $\mathrm{SaN}$ ) and Anger/Surprise (ASu) morphs, which are consistently displaced in the direction of their respective Sadness and Surprise "parents."

\section{DISCUSSION}

Like McKelvie (1995, p. 327), we began with the expectation “[... ] that the effect of inversion would vary with different-expressions
Table 3 | Dimensional-salience parameters $\left(w_{i}\right)$ fitting the group configurations to individual subjects' responses, for the upright and inverted modes of FE presentation.

\begin{tabular}{lllll}
\hline Subject/ & Happy- & Fear/Surprise & Disgust & Anger \\
mode & Sad & (D2) & (D3) & (D4) \\
& (D1) & & &
\end{tabular}

\begin{tabular}{lllll}
\hline \multicolumn{4}{l}{ MO (FEMALE POSER) } & SOLUTION \\
DK upright & 0.105 & 0.113 & 0.144 & 0.130 \\
DK inverted & 0.118 & 0.119 & 0.119 & 0.120 \\
HK upright & 0.122 & 0.116 & 0.118 & 0.118 \\
HK inverted & 0.120 & 0.118 & 0.117 & 0.120 \\
WF (MALE POSER) SOLUTION & & \\
SB upright & 0.122 & 0.120 & 0.119 & 0.116 \\
SB inverted & 0.115 & 0.122 & 0.127 & 0.120 \\
BF upright & 0.118 & 0.115 & 0.127 & 0.123 \\
BF inverted & 0.118 & 0.121 & 0.120 & 0.123 \\
\hline
\end{tabular}

Table 4 | Categorization Index (Cl) values derived from the locations of eight morph stimuli within four-dimensional MDS solutions for the upright and inverted modes of FE presentation.

\begin{tabular}{llllllllll}
\hline Poser & Mode & FSu & DF & DSa & SaN & HN & AH & SuH & ASu \\
\hline MO & Upright & 0.46 & 0.55 & 0.50 & 0.46 & 0.21 & 0.74 & 0.78 & 0.59 \\
(female) & Inverted & 0.51 & 0.47 & 0.42 & 0.36 & 0.16 & 0.83 & 0.84 & 0.57 \\
& both & 0.47 & 0.52 & 0.47 & 0.43 & 0.19 & 0.76 & 0.80 & 0.58 \\
WF & Upright & 0.51 & 0.55 & 0.57 & 0.48 & 0.36 & 0.72 & 0.64 & 0.56 \\
(male) & Inverted & 0.48 & 0.44 & 0.70 & 0.45 & 0.40 & 0.70 & 0.63 & 0.53 \\
& both & 0.51 & 0.50 & 0.64 & 0.48 & 0.37 & 0.70 & 0.65 & 0.56
\end{tabular}

Morphs are 50:50 blends of the following prototype FEs: F - Fear; Su - Surprise; D - Disgust; Sa - Sadness; N - Neutral; A - Anger; H - Happiness.

because they depend differentially on configural information." This follows from two assumptions laid out in the Section "Introduction": first, that inversion selectively impairs the processing of configural relative to featural cues; second, that the relative impact of these types of cues varies from one emotion to another. In particular, we expected the inversion to disrupt discrimination of Disgust, a possible example of a "distributed" configuration (Paramei and Benson, 1998; Bimler and Paramei, 2006). If two FE morphs differ by one containing a larger element of (say) Disgust than the other, then the expected selective disruption of configural cues should cause this difference to contribute less to pairwise similarity when the pair is inverted (i.e., the difference between them should be less apparent). The inversion effect was also expected for Surprise, another candidate for a "configural" expression (Bassili, 1979; Calder et al., 2000).

However, contrary to these expectations, the SDT analysis did not reveal any emotion prototype and its morphs to be more impacted by inversion than the others, moreover showing only an overall trend for $d^{\prime}$ to be slightly lower for inverted than for upright FEs (Figure 2). Reduced discriminability of inverted FEs is in accord with the findings of Narme et al., 2011 (healthy controls data), who conclude that a featural strategy had replaced 


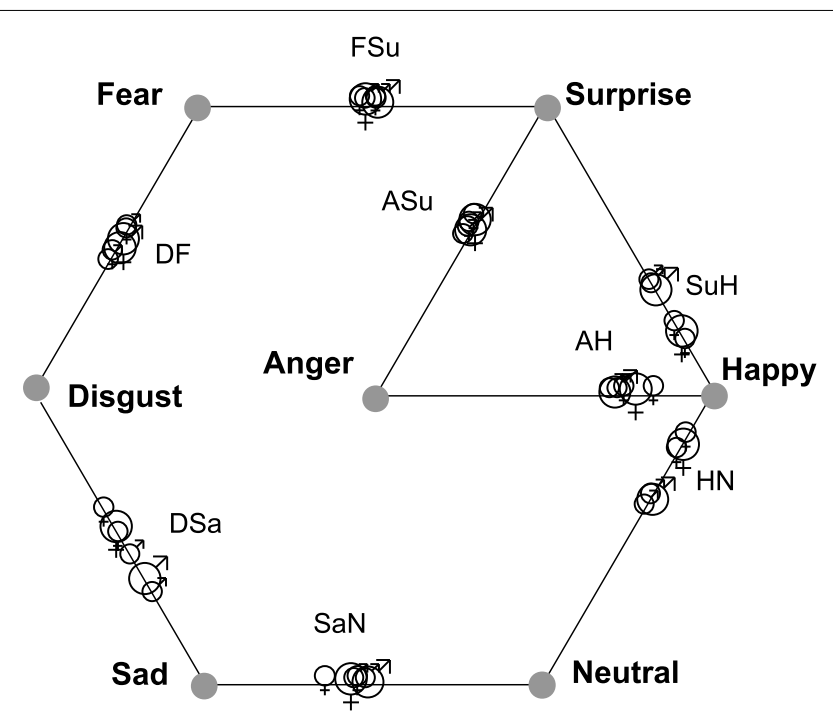

FIGURE 6 |The symbols on each line show the relative proximity (Categorization Index, $\mathrm{Cl}$ ) of one morph FE stimulus to its two emotion "parents" in 4D solutions. $q, \widehat{\delta}$ indicate $\mathrm{Cl}$ for "MO series" (female poser) and "WF series" (male poser) respectively. Smaller symbols indicate $\mathrm{Cl}$ for either upright or inverted solution, larger for the solution aggregating data for both modes of FE presentation.

configural processing, under unlimited exposure. However, those authors additionally found the inversion effect (a significant difference in $d^{\prime}$ between $U$ and I expressions) to be greater for negative emotions, which, it is argued, require greater perceptual processing resources (Prkachin, 2003). We summarized the $\%$ Same frequencies with MDS so as to embed the stimuli within "FE space" and examine expressions in the context of the entire emotional gamut. As shown in the Results, none of the three measures employed to compare solutions - cophenetic and canonical correlations, as well as Procrustes distance - revealed substantial differences between the upright and inverted modes of presentation of discriminated FEs. Also the Individual Differences MDS analysis revealed that inversion made no systematic difference to the perceptual salience of any of the emotion prototypes (i.e., the contribution of the corresponding dimension to inter-stimulus similarity). The "maps" showed little difference between upright and inverted presentation, for either poser.

One explanation for the lack of emotion-specific effect from inversion would be that, in spite of the explicit instruction to compare expressed emotional states, the data reflect the objective similarity between pairs of FEs as visual images, not affective interfaces: it is conceivable that due to the brief exposure of a FE pair, $500 \mathrm{~ms}$, the subjects' judgments of FE "similarity" only tapped into early stages of visual processing, prior to "the locus of emotion perception." In particular, low-level processing might treat images as arbitrary patterns of edges and gray tones (Dailey et al., 2002), devoid of affective connotations, and perform the equivalent of pixel-by-pixel comparison [as in the Principal Components Analysis (PCA) treatment of FEs by Calder et al., 2001] or an edge-emphasizing primal sketch (Marr, 1982).

It is also conceivable, though, that similarity judgments in our exposure conditions were based on more advanced visual processing, i.e., featural information. Lipp et al. (2009), employing a visual search task with the $S / D$ paradigm and an array of nine FEs presented for $6,000 \mathrm{~ms}$ - comparable to the time per stimulus available here - found no effect of inversion on either detection speed or verbal ratings. Indeed, Calvo et al. (2012) provide convincing evidence that, at short stimulus duration, Happy expressions, both upright and inverted, were identified solely via a smiling mouth, this salient feature making it accessible to the visual system. The authors of both studies conclude that, under conditions that preclude/impair configural processing - as is the case with a short stimulus duration efficient decoding of expressions is mediated by feature-based information. This accords with the broadly accepted view that featural cues are relatively insensitive to face orientation, compared to the inversion-related impairment of visual processing of configural cues (Maurer et al., 2002; Carbon and Leder, 2005).

It might seem that the close agreement between the model of the "FE space" obtained here and the maps of the cognitive closeness of various emotional concepts (e.g., Russell, 1980), would be enough evidence that the processing of FE stimuli extended beyond early stages and accessed internal semantic representations of emotions and their relationships. However, there are pragmatic reasons to expect a high degree of parallelism between the pattern of objective similarities among FEs, and the pattern of semantic similarities among the corresponding emotions. Under the lessthan-ideal conditions of real-world interactions (e.g., Smith and Schyns, 2009), where incomplete or degraded information may cause an observer to misidentify the emotional message of a FE, the consequences of a mistake are minimized if it is at least mistaken for a conceptually related emotion. This parallelism was evident when PCA was applied to a database of digitized FE images, treated purely as vectors of gray tones devoid of any cognitive or affective meaning or dependence on image orientation, and yielded factors that were interpretable as intuitive "expression space" axes (Calder et al., 2001).

This is where the CP phenomenon is pertinent. The morphs were constructed so that the difference between (for instance) the absence of a smile (as in the Neutral prototype) and a "half"-smile in the $H N$ morph is equivalent to the difference along the same continuum between $H N$ and a full smile (the Happy prototype). If the geometric FE "maps" accurately reflected physical, objective similarity, then the morphs would be located midway between the prototypal "parents." In fact, the $H N-H$ distance proved to be subjectively smaller than the $N-H N$ distance; the same applying to the $A H$ and the $S u H$ morphs. In consequence, the $H N, A H$, and $\mathrm{SuH}$ morphs were all confused relatively often with the Happiness prototype, and with one another, and all lie close together in every MDS solution we examined, for both stimulus series (see Figures 4 and 5 ).

It follows that the subjective difference produced by shifting the proportions of, e.g., Neutral and Happy depend on the position along that continuum. This non-linear response to varying proportions is a necessary condition of CP (e.g., Calder et al., 1996; Young et al., 1997). As further conditions, the response function should be sigmoidal (and not for instance logarithmic) with the steepest slope at the "Mona Lisa" stimulus, perceived as balanced on the cusp between Neutral and Happy. To establish 
these would require data for additional morphs, spaced more closely.

We can only observe that the "Mona Lisa" stimulus contains substantially less than $50 \%$ of Happiness [cf. the Calvo et al. (2012) blended Neutral (upper face)-Happy expression]. It is tempting to speak of an expression template attuned to the shape of the mouth - a "smile detector" - which saturates at a ceiling level, at quite mild degrees of curvature, and operates to extract the dominant FE feature at an early stage of FE processing. Notably, abundant evidence has accumulated that Happy expressions are processed more rapidly and with greater sensitivity (e.g., Esteves and Öhman, 1993; Leppanen and Hietanen, 2004; Maxwell and Davidson, 2004). Also, recognition of Happy prototypes was better than chance for backward-masked exposures as short as ca. $20 \mathrm{~ms}$ (Maxwell and Davidson, 2004; Milders et al., 2008). Several studies have demonstrated the visual salience of smiling mouth as the diagnostic feature for recognition of Happiness (Smith et al., 2005; Calvo and Nummenmaa, 2008; Schyns et al., 2009; Smith and Schyns, 2009; Calvo et al., 2012).

Other authors have described how heightened discrimination at the "watershed" along a perceptual continuum can arise from general pattern-recognition, feature-integration principles without any special instantiation of "categories" (Ellison and Massaro, 1997; Fiorentini and Viviani, 2009; Calvo et al., 2012), further qualifying the term "CP." The crucial point is that such models require higher levels of processing. Whether the term applied is " $\mathrm{CP}$ " or something else, these non-linear relationships between physical difference and subjective dissimilarities imply that the similarity decisions are indeed accessing some level of semantic processing higher than a basic comparison of gray tones.

Fiorentini and Viviani (2009) found that only discrimination along the Valence axis met all the conditions for CP, i.e., between Happy FEs and others - in agreement with the indications of CP for Happy FEs demonstrated in the present study. The present data point also to signs of CP along the Sad-Neutral ( $S a N)$ and Anger-Surprise (ASu) continua, with the $S a N$ morph perceived as more similar to Sadness and the ASu morph as relatively similar to Surprise. The emerging categorization of Happiness and Surprise is in accord with Smith and Schyns (2009) who found that both expressions are low-spatial-frequency rich and involve the mouth as its diagnostic feature more than other prototype FEs.

It is notable that signs of $\mathrm{CP}$ along a morphed continuum were found for even shorter exposures, $150 \mathrm{~ms}$, in the Same/Different task (Suzuki et al., 2004), and in an identification task for prototype emotions, $200 \mathrm{~ms}$ (Derntl et al., 2009).

Initial light upon the timescale and hierarchy of FE processing - featural vs. configural distinction; visual vs. affective information - was shed by research recording ERPs. In particular,

\section{REFERENCES}

Alvarado, N., and Jameson, K. A. (2002). Varieties of anger. The relations between emotion terms and components of anger expressions. Motiv. Emot. 26, 153-182.

Ashley, V., Vuilleumier, P., and Swick, D. (2004). Time course and specificity of event-related potentials to emotional expressions. Neuroreport $15,211-216$.

Bartlett, J. C., and Searcy, J. (1993). Inversion and configuration of faces. Cogn. Psychol. 25, 281-316.

Bassili, J. N. (1979). Emotion recognition: the role of facial movement and the relative importance of upper and lower areas of the face. J. Pers. Soc. Psychol. 37, 2049-2058.

primary visual processing of a face was found to be defined by the P120/N120, a complex which is too early to be modulated by the emotion category (Campanella et al., 2002). Additionally, the N170 component has been widely regarded as sensitive to faces compared to other objects (Eimer and Holmes, 2002; Ashley et al., 2004). Notably, for inverted faces the N170 component appears later and with lower amplitude. More recent studies report some ERP correlates of emotional content preceding the N170 component, namely a greater frontal positivity around $150 \mathrm{~ms}$ (Eimer and Holmes, 2007) and enhanced posterior negativity for Happy faces around $130 \mathrm{~ms}$ (Schacht and Sommer, 2009). Finally, the emotionrelated differences in facial configuration have been linked to ERP components with a peak at around $300 \mathrm{~ms}$ (Ashley et al., 2004) and a sustained positive wave after $300 \mathrm{~ms}$ (Eimer and Holmes, 2002).

This timescale of FE processing indicated by psychophysiological measures, specifically that the affective content of a FE stimulus is still being processed $300 \mathrm{~ms}$ after its onset, can reasonably be linked to the fact that the indications of CP in the present study were as strong for inverted as for upright FEs (Figure 6, Table 3). It seems that the brief $(500 \mathrm{~ms}$ ) exposure of stimulus pairs in this study disrupted face configural processing and, thus, enabled a "snapshot" in the microgenesis of a process of FE analysis (cf. Carbon and Leder, 2005; Schwaninger et al., 2006). The exposure was conceivably too short for processing to reach completion, i.e., the decoding of configural information used for affective discrimination, but sufficiently long to extract low-level visual pattern information and, beyond that, for quick detection of the salient mouth feature that permitted Valence evaluation of FEs.

We speculate that if pairs had been presented longer, the results would have been more dependent on stimulus orientation. This assumption is indirectly supported by the fact that in studies reporting a $\mathrm{CP}$ effect for discriminated FEs, typical presentation times were longer, $750 \mathrm{~ms}$ (Pollak and Kistler, 2002) or $1000 \mathrm{~ms}$ (de Gelder et al., 1997; Roberson et al., 2007). Further, since at longer exposure times (15 s, McKelvie, 1995; unlimited, Derntl et al., 2009) inverting FEs does not completely disrupt their verbal labeling, the latter authors consider that the effect of FE inversion takes the form of a slowing of configural processing.

\section{ACKNOWLEDGMENTS}

We thank the participants of this study for their time, understanding, and collaborative spirit. The authors are grateful to Sina Gray (neé Williams) for indispensable assistance in carrying out experiments and to Wolfgang Bösche for adapting the software for stimulus generation. Valuable advice on the experimental design from Ehtibar N. Dzhafarov and Hans Colonius are gratefully acknowledged. Helpful comments of two anonymous reviewers are highly appreciated.

Bimler, D., and Kirkland, J. (2001). Categorical perception of facial expressions of emotion: evidence from multidimensional scaling. Cogn. Emot. 15, 633-658.

Bimler, D. L., and Paramei, G. V. (2006). Facial-expression affective attributes and their configural correlates: components and categories. Span. J. Psychol. 9, 19-31.
Calder, A. J., Burton, A. M., Miller, P., Young, A. W., and Akamatsu, S. (2001). A principal components analysis of facial expressions. Vision Res. 41, 1179-1208.

Calder, A. J., Young, A., Perrett, D., Etcoff, N., and Rowland, D. (1996). Categorical perception of morphed facial expressions. Vis. Cogn. 3, 81-117. 
Calder, A. J., Young, A. W., Keane, J., and Dean, M. (2000). Configural information in facial expression perception. J. Exp. Psychol. Hum. Percept. Perform. 26, 527-551.

Calvo, M. G., Fernández-Martín, A., and Nummenmaa, L. (2012). Perceptual, categorical, and affective processing of ambiguous smiling facial expressions. Cognition 125, 373-393.

Calvo, M. G., and Nummenmaa, L. (2008). Detection of emotional faces: salient physical features guide effective visual search. J. Exp. Psychol. Gen. 137, 471-494.

Campanella, S., Quinet, P., Bruyer, R., Crommelinck, M., and Guerit, J.M. (2002). Categorical perception of happiness and fear facial expressions: an ERP study. J. Cogn. Neurosci. 14, 210-227.

Carbon, C.-C., and Leder, H. (2005). When feature information comes first! Early processing of inverted faces. Perception 34, 1117-1134.

Dailey, M. N., Cottrell, G. W., Padgett, C., and Adolphs, R. (2002). EMPATH: a neural network that categorizes facial expressions. J. Cogn. Neurosci. 14, 1158-1173.

de Gelder, B., Teunisse, J.-P., and Benson, P. J. (1997). Categorical perception of facial expressions: categories and their internal structure. Cogn. Emot. $11,1-23$.

Derntl, B., Seidel, E.-M., Kainz, E., and Carbon, C.-C. (2009). Recognition of emotional expressions is affected by inversion and presentation time. Perception 38, 1849-1862.

Diamond, R., and Carey, S. (1986). Why faces are and are not special: an effect of expertise. J. Exp. Psychol. Gen. 115, 107-117.

Eimer, M., and Holmes, A. (2002). An ERP study on the time course of emotional face processing. Neuroreport $13,1-5$.

Eimer, M., and Holmes, A. (2007). Event-related brain potential correlates of emotional face processing. Neuropsychologia 45, 15-31.

Ekman, P., and Friesen, W. V. (1976). Pictures of Facial Affect. Palo Alto, CA: Consulting Psychologists Press.

Ellison, J. W., and Massaro, D. W. (1997). Featural evaluation, integration and judgment of facial affect. J. Exp. Psychol. Hum. Percept. Perform. 23, 213-226.

Esteves, S., and Öhman, A. (1993). Masking the face: recognition of emotional facial expressions as a function of the parameters of backward masking. Scand. J. Psychol. 34, 1-18.

Etcoff, N. L., and Magee, J. J. (1992). Categorical perception of facial expressions. Cognition 44, 227-240.
Farah, M. J., Tanaka, J. W., and Drain, H. M. (1995). What causes the face inversion effect? J. Exp. Psychol. Hum. Percept. Perform. 21, 628-634.

Fiorentini, C., and Viviani, P. (2009). Perceiving facial expressions. Vis. Cogn. 17, 373-411.

Gladstones, W. H. (1962). A multidimensional study of facial expression of emotion. Aust. J. Psychol. 14, 95-100.

Green, D. A., and Swets, J. A. (1966). Signal Detection Theory. New York: Wiley.

Kotsoni, E., de Haan, M., and Johnson, M. H. (2001). Categorical perception of facial expressions by 7month-old infants. Perception 30, 1115-1125.

Kruskal, J. B., and Wish, M. (1978). Multidimensional scaling. Sage University Paper series on Quantitative Applications in the Social Sciences 07-011. Newbury Park, CA: Sage.

Leder, H., and Bruce, V. (2000). When inverted faces are recognised: the role of configural information in face recognition. Q. J. Exp. Psychol. A 53, 513-536.

Leppanen, J. M., and Hietanen, J. K. (2004). Positive facial expressions are recognized faster than negative facial expressions, but why? Psychol. Res. 69, 22-29.

Lipp, O. V., Price, S. M., and Tellegen, C. L. (2009). No effect of inversion on attentional and affective processing of facial expressions. Emotion 9, 248-259.

Marr, D. (1982). Vision: A Computational Investigation into the Human Representation and Processing of Visual Information. San Francisco: Freeman.

Maurer, D., Le Grand, R., and Mondloch, C. J. (2002). The many faces of configural processing. Trends Cogn. Sci. (Regul. Ed.) 6, 255-259.

Maxwell, J. S., and Davidson, R. J. (2004). Unequally masked: indexing differences in the perceptual salience of "unseen" facial expressions. Cogn. Emot. 18, 1009-1026.

McKelvie, S. J. (1995). Emotional expression in upside-down faces: evidence for configurational and componential processing. Br. J. Soc. Psychol. 34, 325-334.

Milders, M., Sahraie, A., and Logan, S. (2008). Minimum presentation time for masked facial expression discrimination. Cogn. Emot. 22, 63-82.

Narme, P., Bonnet, A.-M., Dubois, B., and Chaby, L. (2011). Understanding facial emotion expression in Parkinson's disease: the role of configural processing. Neuropsychologia 49, 3295-3302.
Paramei, G. V., and Benson, P. J. (1998). A facial topology mediates decoding of facial expressions. Perception 27(Suppl.), 132.

Paramei, G. V., Bimler, D. L., and Skwarek, S. J. (2009). "Comparing reaction times and accuracy rates in a same/different task with facial expressions, in Fechner Day 2009," in Proceedings of the 25th Annual Meeting of the International Society for Psychophysics, eds M. A. Elliott, S. Antonijevic, S. Berthaud, P. Mulcahy, C. Martyn, B. Bargery, and H. Schmidt (Galway: National University of Ireland), 251-254.

Parks, T. E., Coss, R. G., and Coss, C. S. (1985). Thatcher and the Cheshire cat: context and the processing of facial features. Perception 14, 747-754.

Pollak, S. D., and Kistler, D. J. (2002). Early experience is associated with the development of categorical representations for facial expressions of emotion. Proc. Natl. Acad. Sci. U.S.A. 99, 9072-9076.

Prkachin, G. C. (2003). The effects of orientation on detection and identification of facial expressions of emotion. Br. J. Psychol. 94, 45-62.

Roberson, D., Damjanovic, L., and Pilling, M. (2007). Categorical perception of facial expressions: evidence for a 'Category Adjustment' model. Mem. Cognit. 35, 1814-1829.

Roberson, D., and Davidoff, J. (2000). The categorical perception of colors and facial expressions: the effect of verbal interference. Mem. Cognit. 28, 977-986.

Roberson, D., Davidoff, J., and Braisby, N. (1999). Similarity and categorization: neuropsychological evidence for a dissociation in explicit categorization tasks. Cognition 71, 1-42.

Russell, J. A. (1980). A circumplex model of affect. J. Pers. Soc. Psychol. 39, 1161-1178.

Schacht, A., and Sommer, W. (2009) Emotions in word and face processing: early and late cortical responses. Brain Cogn. 69, 538-550.

Schwaninger, A., Wallraven, C., Cunningham, D. W., and Chiller-Glaus, S. D. (2006). "Processing of facial identity and expression: a psychophysical, physiological and computational perspective," in Progress in Brain Research: Understanding Emotions, Vol. 156, eds. S. Anders, G. Ende, M. Junghofer, J. Kissler, D. Wildgruber (Amsterdam: Elsevier), 321-343.

Schyns, P. G., Petro, L. S., and Smith, M. L. (2009). Transmission of facial expressions of emotion coevolved with their efficient decoding in the brain: behavioral and brain evidence. PLoS ONE 4:e5625. doi:10.1371/journal.pone.0005625

Shibui, S., Yamada, H., Sato, T., and Shigemasu, K. (2001). The relationship between the categorical perception of facial expressions and semantic distances. Shinrigaku Kenkyu 72, 219-226.

Smith, F. W., and Schyns, P. G. (2009). Smile through your fear and sadness: transmitting and identifying facial expression signals over a range of viewing distances. Psychol. Sci. 20, 1202-1208.

Smith, M. L., Cottrell, G. W., Gosselin, F., and Schyns, P. G. (2005). Transmitting and decoding facial expressions. Psychol. Sci. 16, 184-189.

Stringer, P. (1967). Cluster analysis of non-verbal judgments of facial expressions. Br. J. Math. Stat. Psychol. 20, 71-79.

Suzuki, A., Shibui, S., and Shigemasu, K. (2004). "Temporal characteristics of categorical perception of emotional facial expressions," in Proceedings of the Twenty-Sixth Annual Conference of the Cognitive Science Society, eds K. Forbus, D. Gentner, and T. Regier (Erlbaum), 1303-1308.

Yin, R. K. (1969). Looking at upsidedown faces. J. Exp. Psychol. 81, 141-145.

Young, A. W., Rowland, D., Calder, A. J., Etcoff, N. L., Seth, A., and Perrett, D. I. (1997). Facial expression megamix: tests of dimensional and category accounts of emotion recognition. Cognition 63 271-313.

Conflict of Interest Statement: The authors declare that the research was conducted in the absence of any commercial or financial relationships that could be construed as a potential conflict of interest.

Received: 01 September 2012; paper pending published: 01 October 2012; accepted: 24 January 2013; published online: 14 February 2013.

Citation: Bimler DL, Skwarek SJ and Paramei GV (2013) Processing facial expressions of emotion: upright vs. inverted images. Front. Psychology 4:54. doi: 10.3389/fpsyg.2013.00054

This article was submitted to Frontiers in Cognitive Science, a specialty of Frontiers in Psychology.

Copyright (c) 2013 Bimler, Skwarek and Paramei. This is an open-access article distributed under the terms of the Creative Commons Attribution License, which permits use, distribution and reproduction in other forums, provided the original authors and source are credited and subject to any copyright notices concerning any third-party graphics etc. 


\section{APPENDIX}

SDT analysis enabled us to address a concern that subjects "overlearned" each stimulus pair during the course of 100 trials (despite the intervals separating the 11 sessions of data collection) and came to provide automatic, stereotyped responses (no longer tapping into the perceptual mechanisms that were the target of the research). However, if responses became over-learned, one would expect sensitivity measure $d^{\prime}$ to increase with advancing runs. As Figure A1 shows, there is no evidence of this.

Note also that stereotyped responses, if they became the rule at an early stage of data collection (a predictable Same or Different response for a given pair), would leave no pairs with an intermediate percentage of Same responses; but this degree of polarization is not evident in Figure 3.

As an additional precaution we examined individual pairs; the probability of a Same response generally remained constant over time: it was not a case of an initially stochastic pattern polarizing to deterministic "always Same" or "always Different" patterns. For sufficiently different pairs where this probability remained at nearly 0 from the start of data collection, and for sufficiently similar pairs where the probability remained at 1, over-learning is irrelevant.

As a final precaution, we obtained four-dimensional MDS solutions for the first half of each subject's data (i.e., only 50 presentations of each stimulus pair) for comparison with results based on complete-data. In general Stress $s_{1}$ values were slightly higher for the partial-data solutions (i.e., more random noise) but there was no sign of any systematic changes from excluding the latter 50 trials. For upright stimuli, the cophenetic correlations between complete-data and partial-data solutions were 0.95 for subject BF; 0.95 for SB; 0.96 for DK, and 0.98 for HK. For inverted stimuli the respective correlations were $0.94 ; 0.97 ; 0.98 ; 0.99$.
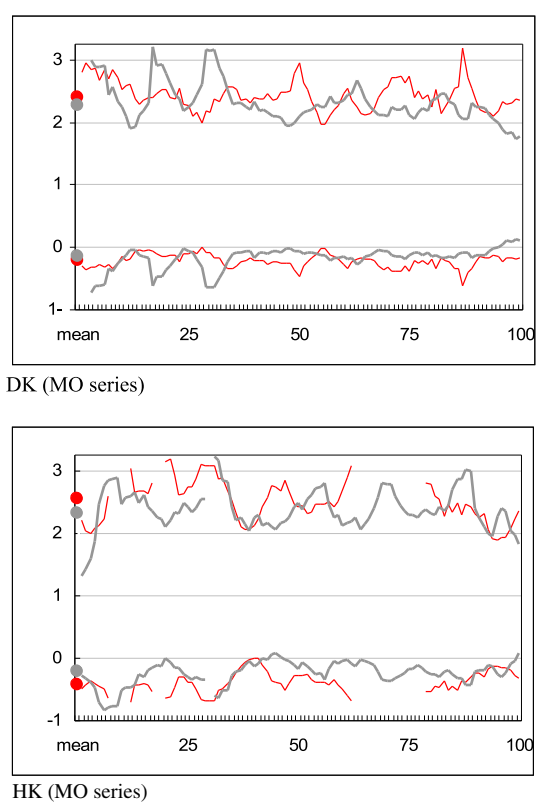

FIGURE A1 | SDT parameters derived from each subject's rates of Same responses to identical- and different-expression pairs. Vertical axis: sensitivity measure $d^{\prime}$ (upper line) and response criterion $C$ (lower line); horizontal axis: experimental run. Left-hand points are mean parameters over all 100 runs. -.-- Upright FEs;

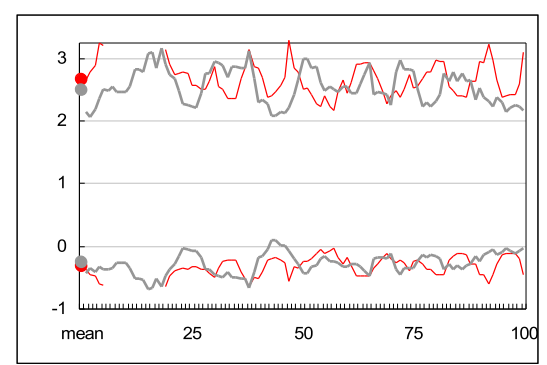

BF (WF series)

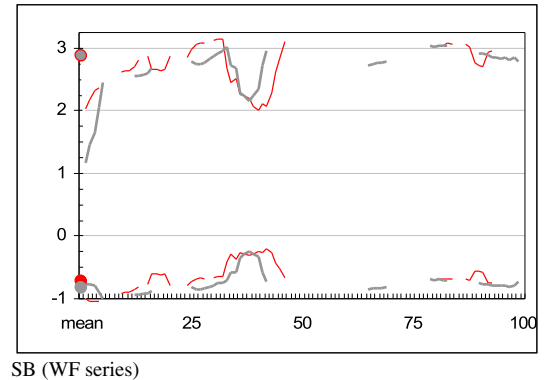

- - Inverted FEs. Values have been smoothed for clarity, by subjecting the numbers of "hits" and "false alarms" to a running mean with a 5-run window before calculating $d^{\prime}$ and $C$. Parameters are not defined for runs in which all identical-expression pairs were identified correctly. 